\title{
The organization and evolution of the Responder satellite in species of the Drosophila melanogaster group: dynamic evolution of a target of meiotic drive
}

\author{
Amanda M Larracuente
}

\begin{abstract}
Background: Satellite DNA can make up a substantial fraction of eukaryotic genomes and has roles in genome structure and chromosome segregation. The rapid evolution of satellite DNA can contribute to genomic instability and genetic incompatibilities between species. Despite its ubiquity and its contribution to genome evolution, we currently know little about the dynamics of satellite DNA evolution. The Responder (Rsp) satellite DNA family is found in the pericentric heterochromatin of chromosome 2 of Drosophila melanogaster. Rsp is well-known for being the target of Segregation Distorter (SD) an autosomal meiotic drive system in D. melanogaster. I present an evolutionary genetic analysis of the Rsp family of repeats in D. melanogaster and its closely-related species in the melanogaster group (D. simulans, D. sechellia, D. mauritiana, D. erecta, and D. yakuba) using a combination of available BAC sequences, whole genome shotgun Sanger reads, Illumina short read deep sequencing, and fluorescence in situ hybridization.
\end{abstract}

Results: I show that Rsp repeats have euchromatic locations throughout the D. melanogaster genome, that Rsp arrays show evidence for concerted evolution, and that Rsp repeats exist outside of D. melanogaster, in the melanogaster group. The repeats in these species are considerably diverged at the sequence level compared to D. melanogaster, and have a strikingly different genomic distribution, even between closely-related sister taxa.

Conclusions: The genomic organization of the Rsp repeat in the D. melanogaster genome is complex it exists of large blocks of tandem repeats in the heterochromatin and small blocks of tandem repeats in the euchromatin. My discovery of heterochromatic Rsp-like sequences outside of D. melanogaster suggests that SD evolved after its target satellite and that the evolution of the Rsp satellite family is highly dynamic over a short evolutionary time scale ( $<240,000$ years).

Keywords: Satellite DNA, Segregation Distorter, Responder, Drosophila, Meiotic drive, Concerted evolution

\section{Background}

Genomes are frequently in conflict with selfish genetic elements that propagate in genomes or populations despite the harm that they cause to the host [1-3]. Genetic elements can range in their degree of selfishness from the expansion of blocks of tandemly repeated satellite DNAs [4] typically found near centromeres and telomeres [5], to the invasive properties of transposable elements

Correspondence: alarracu@bio.rochester.edu

Department of Biology, River campus, University of Rochester, 480 Hutchison Hall, Rochester, NY 14627, USA or the ultra-selfish behavior of meiotic drivers. Meiotic drivers spread in populations by gaining a transmission advantage through gametogenesis [6]. Segregation Distorter $(S D)$ is an autosomal male meiotic drive system in Drosophila melanogaster that has biased transmission while heterozygous females transmit $S D$ fairly to half of their progeny, heterozygous males transmit $S D$ to nearly all of their progeny [7]. SD targets Responder $(R s p)$, a satellite DNA in the pericentric heterochromatin of $2 R[8,9]$. The sensitivity of the Rsp locus to segregation distortion correlates positively with the number

\section{Biomed Central}

2014 Larracuente; licensee BioMed Central. This is an Open Access article distributed under the terms of the Creative Commons Attribution License (http://creativecommons.org/licenses/by/4.0), which permits unrestricted use, distribution, and reproduction in any medium, provided the original work is properly credited. The Creative Commons Public Domain Dedication waiver (http://creativecommons.org/publicdomain/zero/1.0/) applies to the data made available in this article, unless otherwise stated. 
of repeats on $2 R \quad S D$ targets wild-type chromosomes with many $R s p$ repeats $[8,9]$ resulting in wild-type sperm dysfunction through a currently unknown mechanism (reviewed in [10]).

The structure of the Rsp locus is complex: the canonical form of the repeat is a dimer of two related 120-bp repeats referred to as Left and Right Rsp (84\% identical), but the tandemly arrayed canonical repeats are interspersed with more divergent variants of Rsp [11]. At least two additional locations of $R s p$ repeats exist outside of the $S D$ target in $2 R$ pericentric heterochromatin: a cluster of repeats occurs on $3 L$ in cytological band $80 \mathrm{C}$ [12], and a single fragment of a $R s p$ repeat occurs on $2 R$ at cytological band 60A [11].

The evolutionary dynamics of the Rsp satellite are currently unknown. $S D$ is a selfish genetic system specific to $D$. melanogaster. While the divergence between the Right and Left Rsp repeats suggests that the repeat is old, it has never been found outside of D. melanogaster implying that the repeat arose in an ancestor of the melanogaster group and was subsequently lost outside of $D$. melanogaster, or it is rapidly evolving in D. melanogaster. These inferences resulted from studies based on DNADNA hybridization or poorly assembled and fairly low coverage genomes, however, and currently available genomic resources could provide new insights into the evolutionary history of $R s p$ repeat family evolution.

We know little about the evolutionary dynamics of satellite DNAs in general. Many repetitive DNAs undergo concerted evolution [13-15], whereby unequal recombination and/or gene conversion events cause repeats within a species to be more similar to each other than to their homologous repeats between species [15-20]. While concerted evolution is documented among various repetitive DNA sequences, the effect of intragenomic conflict on any particular family of repeats is understudied. While some satellite DNAs are expected to be selfish themselves, the $R s p$ repeats of $D$. melanogaster are instead (or perhaps in addition), the target of a selfish meiotic driver, making these especially interesting repeats to study. An evolutionary genetic analysis of the Rsp family of repeats could reveal important details about the evolutionary history of $S D$, as well as the dynamics of satellite DNAs and the effect of intragenomic conflict on genome evolution.

In this paper, I present an evolutionary genomic analysis of the Rsp satellite in D. melanogaster and the three closely related species of the simulans clade ( $D$. simulans, D. sechellia and D. mauritiana). I combine traditional Sanger sequencing data (from BACs and Whole Genome Shotgun assemblies), Next Generation Sequencing (NGS) data (from genomic Illumina reads), and in situ hybridization to study patterns of Rsp satellite evolution on a short evolutionary time scale. I show that $R s p$ repeats have euchromatic locations throughout the $D$. melanogaster genome, that Rsp arrays show evidence for concerted evolution, and that $R s p$ repeats exist outside of $D$. melanogaster, in species of the melanogaster group. My analyses suggest that $R s p$ repeat family evolution is highly dynamic, and are consistent with the rapid evolution of Rsp in $D$. melanogaster, where it is a target of meiotic drive.

\section{Results and discussion \\ Rsp in D. melanogaster}

I used BLAST to identify Rsp repeats in the WGS assembly of $D$. melanogaster and found hits on nearly every chromosome arm (Figure 1). As expected, large blocks of canonical Rsp repeats defined as the sequences similar to those correlated with the sensitivity to segregation distortion in $\mathrm{Wu}$ et al. [9] occur on chromosome $2 R$ (these repeats in versions of the $D$. melanogaster genome prior to v6.01 were found in ArmU and ArmU extra scaffolds). These repeats correspond to the large block of satellite in the pericentric heterochromatin on chromosome $2 R$. Consistent with previous findings [12], at least one large block of canonical Rsp repeats occurs on chromosome $3 L$. However, I found several small blocks of Rsplike repeats in the euchromatic regions of $\mathrm{X}, 2 \mathrm{R}, 3 \mathrm{~L}$ and 3R (Figure $1 \mathrm{~B}$ and $\mathrm{C}$ ). Interestingly, the largest block of euchromatic repeats is found on chromosome $3 L$, in an intron of the gene Argonaute 3 (Ago3). The Ago3 repeats are canonical $R s p$ repeats (Figure $1 \mathrm{~B})$. The euchromatic Rsp repeats consist of between 1 and 12 repeats which, because the assembly of repetitive sequences tends to collapse repeats with nearly-identical sequences, may be considered estimates of the minimum repeat number. The second largest euchromatic Rsp blocks occur on the $\mathrm{X}$ chromosome. The Rsp-like repeats found on the $\mathrm{X}$ chromosome are not canonical $R s p$ repeats, but divergent copies of a $R s p$ family repeat (hereon referred to as $R l X$ for $R s p$-like on $X$; $P$-value from permuted alignments with $D$. melanogaster canonical $R s p<10^{-4}$; Figure 1B). There are three clusters of interspersed $R l X$ repeats in cytological band $4 \mathrm{C}$ on the $\mathrm{X}$ chromosome (occurring within a $150 \mathrm{~kb}$ interval). The three clusters span $715 \mathrm{bp}, 120 \mathrm{bp}$ and $1430 \mathrm{bp}$, respectively, with the largest cluster occurring within $1 \mathrm{~kb}$ of the gene CG12688. I compared all individual WGS reads matching canonical $R s p$ to estimate genome-wide variability in $R s p$ repeat sequence. Overall, individual reads matching the Left and Right canonical Rsp sequences from across the genome shared $85.8 \%$ and $87.6 \%$ identity, indicating that there is considerable variability in canonical $R s p$ repeats in the genome.

Because during genome assembly, reads from identical or nearly identical sequences may be collapsed into a single sequence or mapped to the wrong location, repetitive regions of the genome are often misassembled [21]. This makes it difficult to reliably compare repeats in 


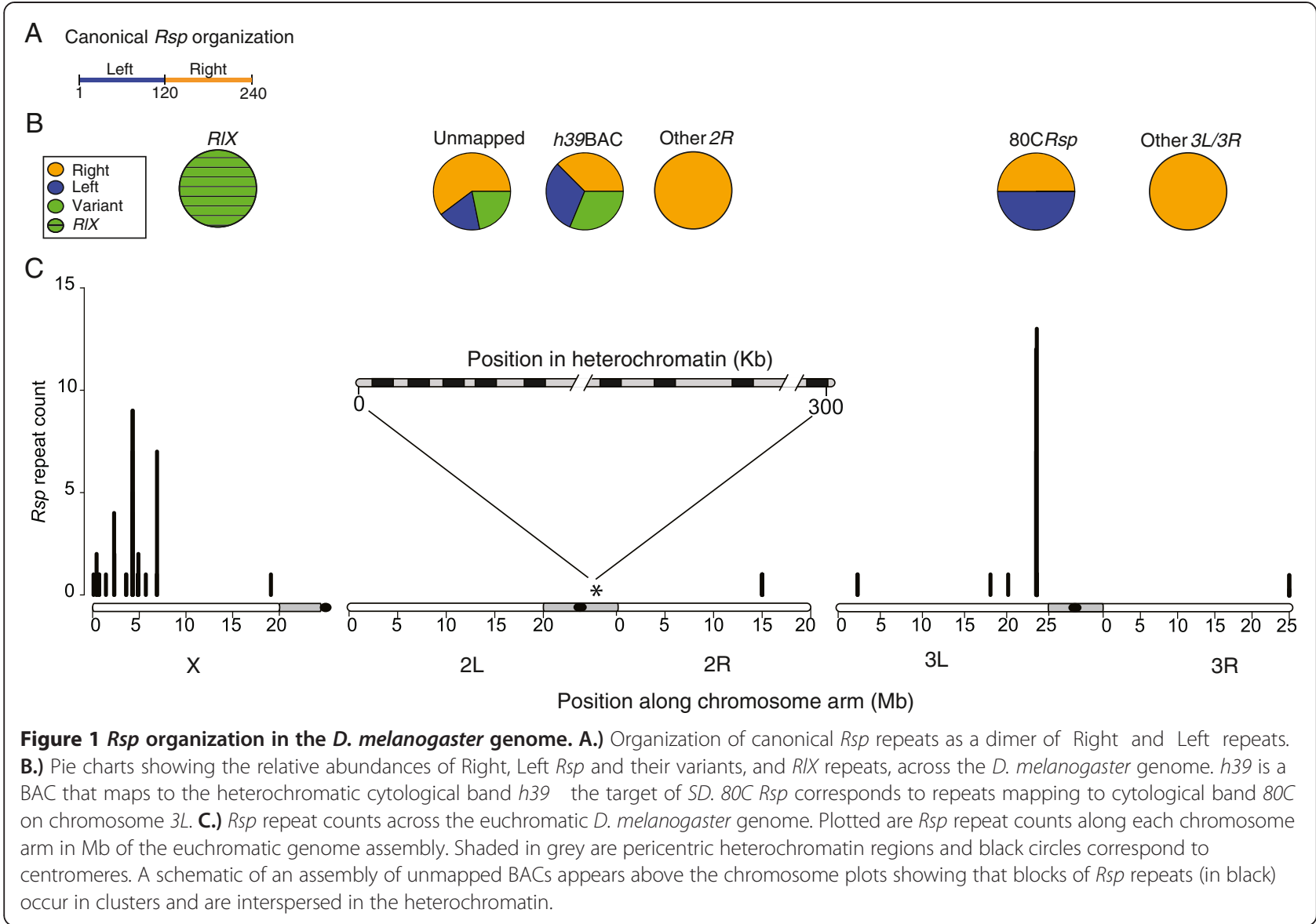

different regions of the WGS assembly. To compare repeats between regions of the genome, I instead used BLAST to identify Rsp repeats in sequenced BACs mapping to known genomic locations. I obtained hits on BACs that map to euchromatic and heterochromatic locations in the genome, including the $2 R$ heterochromatic division called $h 39$, the target of $S D$ [8]. The BAC categorized as $h 39$ (AC246306.1) contains clusters of Bari-1 repeats that define the distal boundary of the Rsp locus at $h 39$ [22], (Additional file 1: Table S1). Within arrays (defined here as tandem blocks of Rsp repeats on nonoverlapping BACs), I categorized repeats into the canonical Left repeats, canonical Right repeats, and variants of the canonical Rsp repeats. Among BACs that are unmapped and likely correspond to pericentric heterochromatin, there is less variability within Left (89.5 percent identity, 95\% C.I. 79.5-100: Table 1) and Right (90.4 percent identity, 95\% C.I. 82.3-100; Table 1) than between Left and Right repeats (82.4 percent identity, 95\% C.I. 76.0-89.8; Table 1). The repeats that map to $h 39$ (the target of $S D$ ), appear more similar (i.e. fewer differences among repeats within the array) than the unmapped reads (Table 1 ). The repeats in the $3 L$ cluster are the most similar of the main blocks of Rsp repeats (Table 1).
The $R l X$ repeats are 52.5-57.7 percent identical (\%ID) to the consensus canonical Rsp sequences over their entire length but well conserved over bases 57110 in the consensus canonical $R s p$ sequences ( $R l X$ vs. Left $R s p$ is 76.7-79.1\%ID; $R l X$ vs. Right $R s p$ is $79.1-86 \% \mathrm{ID})$. Moschetti et al. [12] posited that the original canonical Rsp repeats were identified from a clone that actually maps to $3 L$ instead of $h 39$. Consistent with this idea, $R s p$ repeats found at $80 \mathrm{C}$ on chromosome $3 L$ are primarily the canonical Rsp sequences, whereas repeats found at h39, and unmapped BACs are a mix of canonical $R s p$ and their variants, where variants are

Table 1 Percent identity within and between canonical $R s p$ repeat types, for BACs from unmapped regions, $h 39$ (target of SD), and $3 L$

\begin{tabular}{llll}
\hline BAC location & \multicolumn{2}{l}{ Within-repeat type \%ID } & $\begin{array}{l}\text { Between-repeat } \\
\text { type \%ID } \\
\text { Left-right }\end{array}$ \\
\cline { 2 - 3 } & Left & Right & $82.4(76.0-89.8)$ \\
\hline Unmapped & $89.5(79.5-100)$ & $90.4(82.3-100)$ & $81.5(80.6-82.3)$ \\
h39 & $99.6(99.1-100)$ & $92.1(84.1-100)$ & 81.51 (76.2-91.9) \\
\hline 3L & $96.5(93.0-100)$ & $90.1(94.5-100)$ & 79.4 \\
\hline
\end{tabular}

Canonical repeats have the little variation on $3 L$ and at $h 39$, but are highly variable on the unmapped BAC. Variability between Left and Right canonical repeats does not differ between regions. 
defined as repeats that fall between 79.5\%-90\% identical to right or left canonical Rsp repeats (Figure 1B).

A neighbor-joining tree constructed from $R s p$ repeats found in clusters on different BACs confirms that repeats within an array tend to be more similar to each other than repeats outside of an array (Figure 2), a pattern consistent with a history of concerted evolution. Surprisingly, for some repeat clusters, it appears that there is even exchange between arrays of repeats in different genomic locations and perhaps even different chromosome arms (between unmapped BACs whose likely location is chromosome $2 R$ and $3 L$ BACs; Figure 2). It is however possible that the unmapped BACs map to pericentric heterochromatin on $3 L$ instead of $2 R$, however if this were the case, this result still demonstrates exchange between distinct clusters of repeats (no portion of Ago 3 occurs on this unmapped BAC). Unfortunately, I was unable to conclusively determine the genomic location of the unmapped BACs [23], (Additional file 1: Figure S1). The Rsp repeats found on BACs mapping to $2 R$ (at $h 39$ ) and $3 L$ are very similar [23] (Additional file 1: Table S2), consistent with previous observations [24]. While this may be evidence for rare interchromosomal exchange between $2 R$ and $3 L$, such exchange has not been documented for other repeat families in Drosophila. Alternatively, selective sweeps involving the $2 R$ pericentric heterochromatin could also cause repeats on different chromosomes to be more closely related to each other than nearby repeats, as the different chromosomes would have the same recent common ancestor [24].

\section{Rsp family repeats in simulans clade species}

Although the Rsp satellite has not been described outside of $D$. melanogaster [9,24,25], I found repeats similar to Rsp in each species of the simulans clade, and in $D$. erecta and D. yakuba (hereon referred to as Rsp-like; [23], Additional file 1: Table S3). I used BLAST to identify sequences related to the $D$. melanogaster Rsp repeat in the WGS assemblies of $D$. sechellia, $D$. simulans, $D$. erecta and $D$. yakuba ( $P$-values from permuted alignments with $D$. melanogaster canonical Rsp are $<10^{-4}$ for each species). The repeat unit of Rsp-like is larger than canonical Rsp: Rsp-like is $\sim 160 \mathrm{bp}$ in the simulans clade and $173 \mathrm{bp}$ in D. erecta, whereas canonical Rsp in D. melanogaster is $\sim 120 \mathrm{bp}$. Throughout the paper, only the $\sim 120 \mathrm{bp}$ homologous to canonical Rsp is analyzed. Interestingly, $D$. erecta Rsp-like repeats appear to have a dimeric structure analogous to the Left and Right repeats of $D$. melanogaster. I refer to the D. erecta repeats as Rsp-like-1 and Rsp-like-2 (there is $9.5 \%$ divergence between the pairs) to avoid confusion with the Left and Right repeats of D. melanogaster because the dimeric structures appear independently derived.

Because repetitive sequences are often underrepresented and misassembled in traditional WGS assemblies $[21,26,27]$, I also queried the reads of Illumina NGS datasets in D. melanogaster and species of the simulans clade $D$. simulans, D. sechellia and D. mauritiana for Rsp sequences. Using the consensus of Rsp-like sequences as references, I identified Rsp and Rsp-like repeats among the NGS reads of $D$. melanogaster, $D$. sechellia, $D$. simulans, and D. mauritiana using Bowtie2 (KP016744KP016746). I collected all unique Rsp sequences (individual repeat units) by constructing de novo assemblies of all canonical Rsp and Rsp-like reads for each species (see Methods; Table 2). Species vary widely in their number of unique repeats: $D$. sechellia, in particular, has an order of magnitude more unique repeats than the other species (Table 2).

Because several indels differentiate repeats within and between species, I constructed a neighbor joining tree based on distance using a model that considers adjacent indels as a 5th nucleotide state. This tree was constructed for unique Rsp and Rsp-like sequences (excluding $R l X$ repeats and partial repeat units) in D. melanogaster and species of the simulans clade with D. yakuba as the outgroup [23]. The tree topology reveals that Rsp-like sequences in D. simulans, D. sechellia and D. mauritiana are very similar (Figure 3). The divergence between the canonical D. melanogaster Left and Right Rsp (79-82\% ID) implies that the repeat family originated before the speciation of melanogaster group species, assuming a molecular clock. However, the Rsp repeats in D. melanogaster form a monophyletic group, whereas the Rsp-like repeats of the simulans clade species are intercalated throughout the tree (Figure 3). I also inferred the best maximum likelihood tree for all unique Rsp and Rsp-like sequences and found the same pattern [23], (Additional file 1: Figure S2). The canonical D. melanogaster Rsp and simulans clade species Rsp-like repeats are highly similar in the first $28 \mathrm{bp}$ and the last $66 \mathrm{bp}$, but difficult to align in the middle. I am therefore uncertain of the length of the internal branch leading to the D. melanogaster repeats (Figure 3). Taken together, the excess divergence and monophyly of Rsp repeats on the D. melanogaster branch implies that there has been accelerated evolution in this lineage, where it is the target of $S D$.

\section{Relationship between heterochromatic and euchromatic repeats}

In addition to the heterochromatic Rsp-like repeats, the WGS assemblies of $D$. sechellia and $D$. simulans include the euchromatic $R l X$ repeats. To examine the relationship between the heterochromatic Rsp and Rsp-like repeats and the euchromatic $R l X$ in D. melanogaster, D. simulans, $D$. sechellia, D. mauritiana, D. erecta and D. yakuba, I constructed phylogenies using MrBayes. To test for concerted evolution of the euchromatic $R l X$ repeats, I selected three $R I X$ repeats occurring in orthologous positions upstream of the X-linked gene CG12688 for D. melanogaster, D. 
0.1

\begin{tabular}{|c|}
\hline $\begin{array}{r}2 \mathrm{R} \\
-\quad 60 \mathrm{~A} \\
\end{array}$ \\
\hline $\begin{array}{l}\text { Unmapped } \\
\text { BAC_U } \\
\text { Other_U }\end{array}$ \\
\hline $\begin{aligned} & 3 \mathrm{~L} \\
- & 80 \mathrm{C} \\
- & 66 \mathrm{~A} \\
- & 62 \mathrm{C}\end{aligned}$ \\
\hline $\begin{array}{rl} & \mathrm{X} \\
= & 4 \mathrm{~B}-\mathrm{C} \\
& 20 \\
\mathrm{~B} & 3 \mathrm{~F} \\
\text { X unknown }\end{array}$ \\
\hline
\end{tabular}

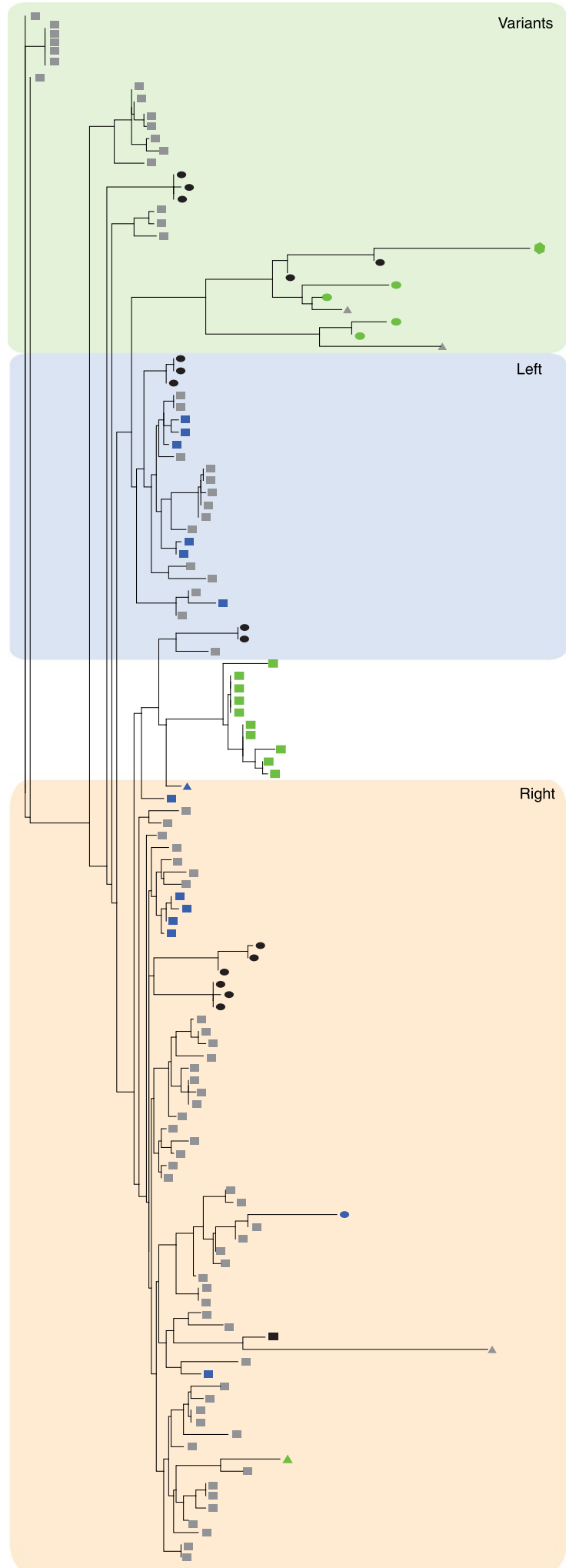

Figure $2 D$. melanogaster Rsp repeat clusters across the genome. Plotted is a neighbor-joining tree of Rsp repeats on BACs across the genome. BACs are color-coded according to location. 
Table 2 Number of unique Rsp or Rsp-like repeats in $D$. melanogaster or the simulans clade, respectively

\begin{tabular}{ll}
\hline Species & Unique repeats $^{\mathbf{a}}$ \\
\hline D. melanogaster & 316 \\
D. simulans & 164 \\
D. sechellia & 1738 \\
D. mauritiana & 32 \\
\hline
\end{tabular}

${ }^{a}$ The number of unique Rsp or Rsp-like repeats extracted from the de novo assemblies of NGS reads (see Methods).

sechellia and $D$. simulans. The tree suggests that the $D$ melanogaster canonical Rsp repeats are indeed monophyletic and evolving rapidly. The tree topology indicates that the Rsp-like repeats of the simulans clade groups with canonical Rsp repeats of D. melanogaster, but with low posterior probability (60\%; Figure 4 ). Comparing overall percent identity, the $R l X$ repeats are more similar to the Rsp-like repeats of the simulans clade than canonical $D$. melanogaster Rsp repeats: whereas $R l X$ to Rsp-like percent identify varies between $75.6-80.7 \%, R l X$ to $R s p$ (canonical) only varies from $55.6-61.5 \%$ [23], again suggesting that canonical Rsp repeats evolve rapidly in $D$. melanogaster.
Although it is possible that canonical Rsp is an old repeat that was lost in the simulans clade, the tree topology suggests that this is not the case (Figure 4). The polytomy between the simulans clade $R l X$ repeats, the $D$. melanogaster $R l X$ repeats and the heterochromatic Rsp and Rsp-like repeats of D. melanogaster and the simulans clade (Figure 4) may be influenced by gene conversion events between $R l X$ repeats and canonical Rsp repeats in D. melanogaster [23]. A comparison of the three tandem $R l X$ repeats in orthologous positions on the X chromosome (in cytological band 4C upstream of CG12688) revealed a pattern of concerted evolution: repeats within a species are more similar than repeats at the orthologous positions between species (Figure 4).

\section{Dynamic genomic distribution of Rsp-like repeats}

The WGS assembly and NGS reads cannot give a complete picture of the satellite DNA distribution in these genomes because of assembly difficulties in heterochromatin. To determine the large-scale organization of the Rsp repeats in these species, I used FISH on mitotic chromosomes from larval neuroblasts using probes specific to

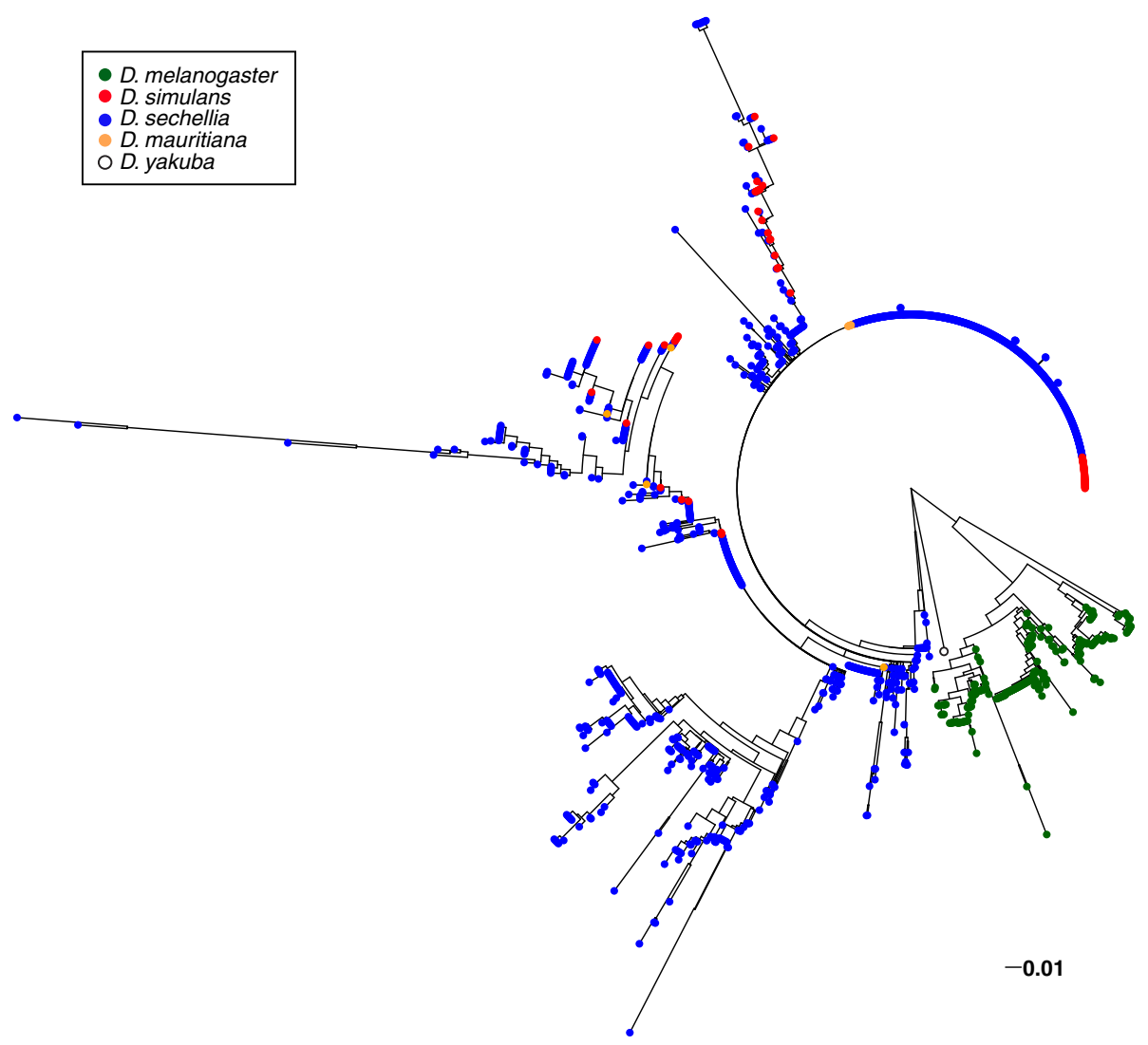

Figure 3 Neighbor joining tree of unique Rsp sequences in D. melanogaster and Rsp-like sequences in species of the simulans clade with $D$. yakuba as the outgroup suggests that $R s p$ evolves rapidly in $D$. melanogaster. These unique sequences were compiled from the NGS reads in each species. The Rsp-like sequences of D.simulans (red), D. sechellia (blue) and D. mauritiana (orange) are interleaved throughout the tree whereas the canonical Rsp in D. melanogaster (green) forms its own clade. 


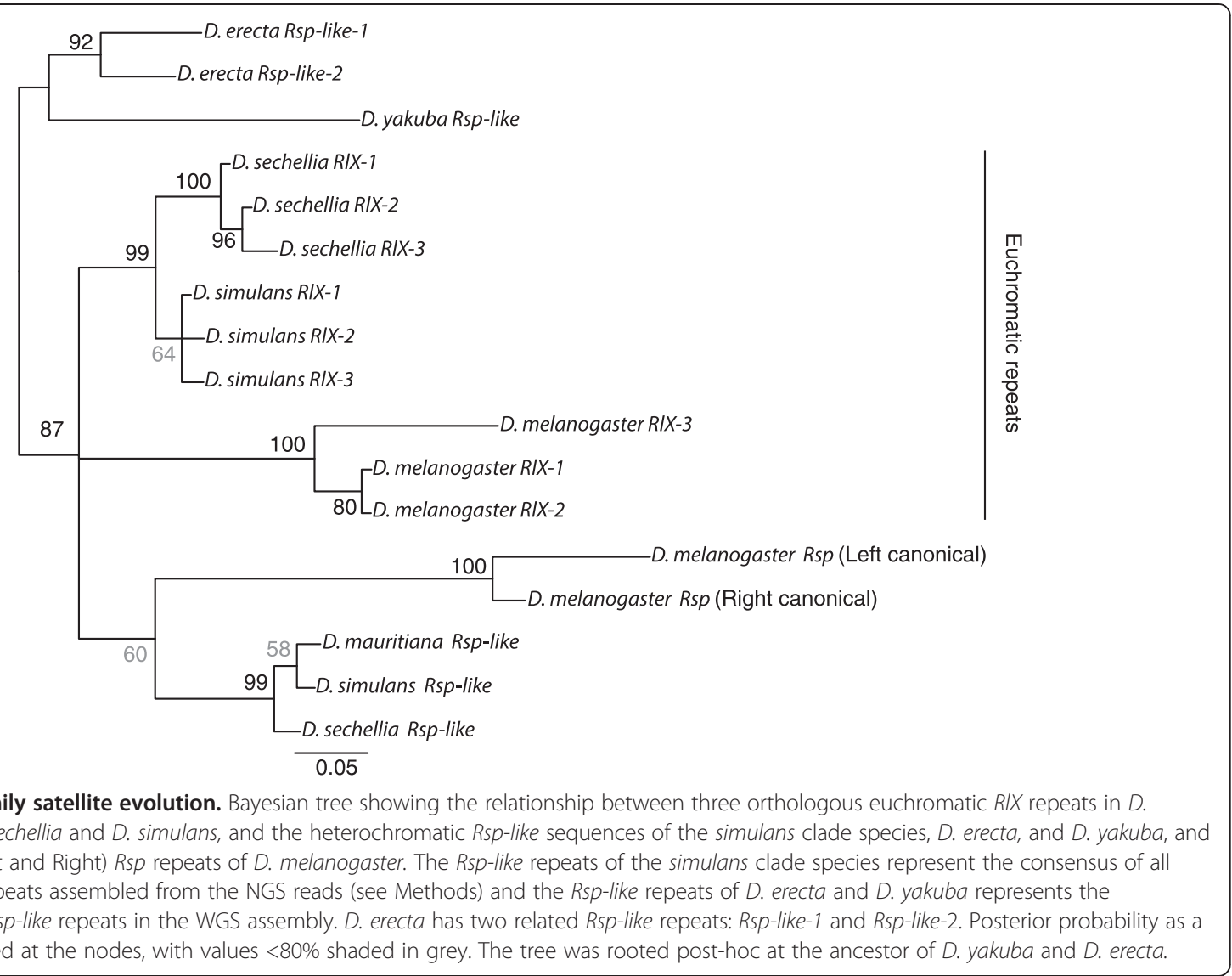

D. melanogaster Rsp (for D. melanogaster FISH) or D. sechellia Rsp (for D. sechellia, D. simulans and D. mauritiana FISH). Small euchromatic satellite repeat islands are not detectable at this resolution, instead the FISH reveals the locations of large blocks of tandem satellite repeats. I discovered that the amount and distribution of Rsp-like repeats varies between the species. Rsp-like repeats exist as large blocks of satellite DNA in D. sechellia and $D$. simulans but not $D$. mauritiana (they are not detectable using FISH on mitotic figures; these repeats are also not detectable in D. yakuba, data not shown). The D. melanogaster probe does not cross hybridize with simulans clade species and the $D$. sechellia probe does not cross hybridize with $D$. melanogaster. Furthermore, the genomic location of Rsp-like satellite blocks has changed dramatically between species. Whereas in D. melanogaster, the Rsp satellite is located in the pericentric region of $2 R$, in $D$. sechellia the Rsp-like satellite is in the pericentric region of $2 R, 3 R$ and $3 L$, and in D. simulans, the Rsp-like satellite occurs only at the base of the $\mathrm{X}$ chromosome (Figure 5). The chromosome arms of these species are homologous and have extremely high degrees of synteny [28] they appear to mostly differ at a gross scale in their distribution of satellite repeats.

\section{Conclusions}

We currently know little about the evolution of satDNA, despite that it can make up a significant fraction of eukaryotic genomes [29]: $>50 \%$ of the genome in kangaroo rats [30] and tenebrionid beetles [31]. Because of its ability to spread in genomes without offering any benefit to the host, satDNA has been considered selfish, junk DNA [1-3]. However, subsequent work in evolutionary, molecular, cellular, and cancer biology has converged on the idea that the heterochromatic fraction of the genome, including satDNAs, has important functional consequences [32-46]. While some blocks of satellite DNA themselves may exhibit meiotic drive, or biased transmission through gametogenesis [6] in females [47,48], the topic of this study the Responder ( $R s p$ ) satellite is a instead the target of meiotic drive in male D. melanogaster [7]. My discovery of highly dynamic evolution in the Rsp satellite family offers a model system to study the evolutionary dynamics of satDNA and the genetic conflict surrounding this enigmatic compartment of the genome.

Most of what we know about satellite DNA dynamics is at the resolution of large blocks of satellite DNA on chromosome arms. Assembly issues with repetitive DNA have stymied our understanding of satellite 


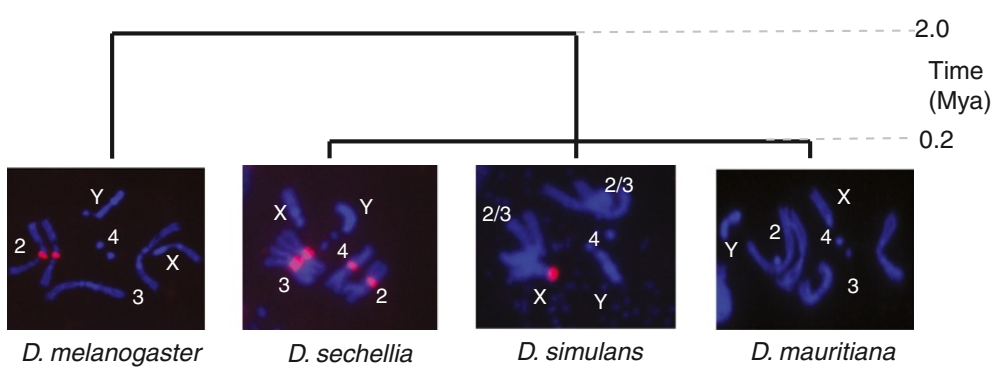

Figure 5 FISH showing Rsp satellite blocks in D. melanogaster and species of the simulans clade. Rsp and Rsp-like repeats are evolutionarily dynamic at the chromosome level.

DNA evolutionary dynamics at the genomic level [21]. Using information from the physical map (e.g. BACs $[27,49])$ and deep Illumina sequencing in combination with FISH offers a higher resolution image of satellite DNA evolution within and between species. My analysis of the Rsp satellite DNA family demonstrates that Rsp: 1) exists outside of the large block of pericentromeric satellite in locations across the D. melanogaster genome; 2) exists in species of the melanogaster group; 3) shows evidence for concerted evolution; and 4) is evolutionary dynamic in its abundance and genomic distribution over short evolutionary time scales.

\section{Euchromatic satellite repeat islands}

The Rsp satellite is a particularly interesting satellite DNA because it is the target of the $S D$ meiotic drive system in D. melanogaster. Moschetti et al. [12] showed using FISH on polytene chromosomes that a small block of Rsp exists on chromosome $3 L$, outside the region associated with $S D$ (at heterochromatic division $h 39$ on $2 R$ ). I show here that in addition to the block of repeats on $3 L$, Rsp family repeats exist in euchromatic satellite repeat islands in locations across the $D$. melanogaster genome, most notably on $3 L$ and the $\mathrm{X}$ chromosome ( $R l X$ repeats). Variable tandem repeats in several taxa have been considered as possible sources of gene regulatory variation (reviewed in [50]). It is possible that these Rsp repeat islands have some regulatory role in the expression of nearby genes or local chromatin condensation: in some cases they occur in or near genes in D. melanogaster. Similar to the genomic distribution of Rsp, short, euchromatic blocks of up to 5 tandem repeats were recently reported clustering in or near genes for satellites of the 1.688 family [14] in D. melanogaster.

The largest euchromatic Rsp islands are on $3 L$ and the $\mathrm{X}$ chromosome. The $\mathrm{X}$ chromosome has a special role in the $S D$ system: escapers from $S D$-mediated meiotic drive have biased sex ratio [51], and X-linked suppressors of $S D$ segregate at high frequencies in natural populations $[52,53]$. Some X chromosomes therefore seem to offer a protective effect against $S D$. Factors that suppress $S D$ map to at least three regions of the X chromosome [54], and some of these intervals contain $R l X$ repeats. It will be interesting to determine if the $R l X$ repeats are involved in suppression of the $S D$ system. One recently proposed hypothesis for the molecular mechanism of segregation distortion suggests that repeat-associated small interfering RNAs (rasiRNAs) corresponding to Rsp are necessary for proper packaging of the satellite during spermiogenesis, and that $S D$ interferes with the production or localization of these rasiRNAs [10,55,56]. Many satellite DNAs in D. melanogaster [37] and other taxa $[34,57,58]$ are transcribed and processed into small RNAs, including Rsp ([59]; Larracuente, unpublished). One possibility is that these euchromatic Rsp satellite repeat islands correspond to Rsp rasiRNA-producing clusters.

\section{Dynamic satellite DNA evolution on small time scales}

$S D$ is a melanogaster-specific drive system $[60,61]$, but the history of the Rsp satellite has been unclear. While divergence between the Left and Right canonical Rsp repeats suggests that the repeat is old, the repeats had not been reported outside of $D$. melanogaster previously [9], although some studies indicated that divergent copies of the repeat may exist in D. simulans [24,25]. My results demonstrate conclusively that Rsp-like sequences exist in abundance in the closely-related species of the simulans clade, adding important insight into the evolution of the $S D$ system $S D$ arose in D. melanogaster in a background that already had $R s p$ repeats to target. The $D$. melanogaster canonical Rsp is highly divergent compared to the Rsp-like repeats of the simulans clade species (e.g. 56-63\% ID) and monophyletic, suggesting that $R s p$ has accelerated evolution in D. melanogaster. What might drive the rapid evolution of this repeat family in D. melanogaster? One possibility is that the mutation rate is unusually high for $D$. melanogaster Rsp compared to the Rsp-like repeats in the simulans clade species. An alternative explanation is that in D. melanogaster, segregation distortion against the canonical Rsp repeats by $S D$ creates selection pressure to diverge from the target sequence. At present, we do not know the molecular 
mechanism of distortion, or what feature of the Rsp locus makes it a target (i.e. if it is sequence-specific). Efforts to compare the detailed evolutionary history of $R s p$ family repeats in the melanogaster group are underway.

Comparing Rsp repeat sequences within and between species revealed that Rsp and Rsp-like sequences show at least two lines of evidence for concerted evolution: 1) repeats within the $D$. melanogaster genome are more similar within a genomic cluster than between genomic clusters; and 2) $R l X$ repeats between $D$. melanogaster and species of the simulans clade are most closely related to nearby repeats within each species than to repeats at orthologous positions between species.

At least in part due to the mutational properties of repetitive DNA [62], the turnover in satellite DNAs between closely-related species can be extreme $[5,63]$ and in some cases may contribute to genetic incompatibilities between species [5,38]. On a genome-wide level, Rsp family evolution is highly dynamic. In the time since $D$. sechellia, D. simulans and D. mauritiana diverged approximately 240 Kya [64] the Rsp-like satellite has dramatically changed its genomic distribution. In $D$. melanogaster, large blocks of the Rsp satellite occur in the pericentric heterochromatin of $2 R$, in $D$. sechellia, the Rsp-like satellite expanded and occurs in the pericentric heterochromatin of $2 R, 3 L$ and $3 R$, in $D$. simulans, the Rsp-like satellite only occurs at the base of the $\mathrm{X}$ chromosome and the Rsp-like satellite is undetectable at the chromosome level in D. mauritiana and $D$. yakuba. Rsp repeat copy number is highly polymorphic within D. melanogaster [65] this polymorphism is directly related to $S D$, as large blocks of satellite confer sensitivity to segregation distortion [8,9]. The Rsp-like repeats in non-melanogaster species are presumably not targets of a meiotic drive system. It will be interesting to compare the population genetics of $R s p$ between species where it is and is not a target of meiotic drive.

\section{Methods}

Querying Whole Genome Shotgun (WGS), BAC assemblies, and reads

D. melanogaster reads were downloaded from the NCBI Trace Archive; BACs [27,49] and WGS contigs [66] were downloaded from Genbank. Rsp repeats were found in the D. melanogaster WGS genome assembly (version 6.01), BACs and NCBI Trace Archive using local basic local alignment search tool (BLAST) searches. Individual traces from four BACs (AC246323.1, AC246299.1, AC007548.10, and AC009843.9; [23], Additional file 1: Figure S1) were obtained from Sue Celniker and Kenneth Wan. An iterative BLAST search protocol was used to gather all sequences matching Rsp. Initial BLAST searches were performed using Rsp sequences deposited in Genbank as queries. To capture as much variation in Rsp sequence as possible, and to recover more divergent Rsp sequences, several BLAST iterations were performed in which subsequent BLAST searches used incrementally refined lists of the hits from previous BLAST searches as queries. This iterative process ended when no additional significant hits were obtained. BLAST hits with an $e$-value $>0.1$ and a length less than $30 \mathrm{bp}$ were excluded from the analysis. BLAST alignments for hits with an $e$-value $>.001$ and length $<50$ were inspected by eye. Redundancies between sequences were removed using custom Perl scripts. Alignments of Rsp sequences were made using BWA-SW [67]. Pairwise percent identity between Rsp repeats was calculated in Geneious (version 6.1.7, created by Biomatters; http://www.geneious.com; [68] and the mean and 95\% confidence intervals of pairwise percent identity were calculated in R. The same procedure was used to query the WGS assemblies of $D$. sechellia [28], D. simulans [69], $D$. erecta [28], and D. yakuba [28]. To determine that Rsp and Rsp-like repeats are indeed related sequences, the nucleotides of each repeat sequence were randomly shuffled and percent identity was re-calculated for pairwise alignments using custom perl scripts. To create a distribution of percent identity based on nucleotide composition, 10,000 permutations were completed for each sequence and P-values were obtained using the empirical cumulative distribution function for each set of permuted alignments (in R).

\section{Querying Next Generation Sequencing (NGS) reads}

Illumina GAIIX paired end reads from $D$. melanogaster (SRR060098; [70]), D. simulans (SRR520350; [69]), D. sechellia (SRR869587; [64]) and D. mauritiana (SRR483621; [64]) were downloaded from the NCBI s SRA (http://www. ncbi.nlm.nih.gov/sra). NGS reads were trimmed of adapters and low quality bases using Trim Galore (version 0.2.8; Babraham Bioinformatics http://www.bioinforma tics.babraham.ac.uk/projects/trim_galore/). Using Bowtie2 [71], the trimmed reads were mapped to consensus Rsp sequences (from the iterative BLAST procedure described above) that capture variation within and between species from WGS and BAC sequences. The alignments were analyzed using Samtools-0.1.18 [72]. To create a list of unique Rsp sequences for each species, de novo assemblies were constructed using the mapped reads extracted from SAM files created from Bowtie2. ABySS (version 1.3.6; [73] was used for the de novo assembly with the following parameters: $k=64 ; s e ; \mathrm{m}=30 ; l=64$. The unique contigs were parsed for individual Rsp sequences using BLAST to the original query Rsp and custom Perl scripts (e.g. a 400bp contig was split into three individual Rsp repeats as determined by BLAST to a consensus Rsp sequence). Some assembled repeat units were less than the canonical repeat unit length ( 120 bp). Because some of these shorter units represent true fragmented repeats and some are expected to be fragments of unique repeats that the de novo 
technique couldn $t$ complete (for reasons such as insufficient read depth or sequencing error), repeats with identical sequence but length variants were merged. Specifically, short, overlapping fragments with identical sequence and overhangs (non-overlapping sequence) of $<50 \%$ of the total fragment length were merged into a single unique repeat. The list of unique $R s p$ repeats in each species was imported into Geneious (version 6.1.7 [68] to create and edit alignments.

\section{Trees}

Neighbor-joining trees (Saitou and Nei 1987) were constructed using bionj with the ape package in R. Blocks of adjacent indels were included in the model using the setting model = indelblock and bootstrapping was done using the boot.phylo function in the ape package $(B=100)$. Bayesian inference of the phylogenetic relationships between the $R l X$, Rsp-like and canonical Rsp repeats was performed using MrBayes [74] using a GTR nucleotide substitution model with Gamma rate variation. The Rsp-like sequences of the simulans clade species were consensus sequences (GenBank accession numbers: KP016744, KP016745, KP016746) of the de novo contigs assembled from each species NGS reads. The Rsp-like repeats of D. yakuba and D. erecta are consensus sequences from the BLAST of WGS assemblies. The $R l X-1, R l X-2$ and $R l X-3$ are orthologs of three tandemly-repeated $R I X$ sequences from cytological band $4 \mathrm{C}$ in the region $\sim 1 \mathrm{~kb}$ upstream of CG12688.

Maximum likelihood inference of the best tree for the unique Rsp sequences from the NGS reads of $D$. melanogaster, D. sechellia, D. simulans and D. mauritiana was performed in RAxML v7.4.2 [75] using the CIPRES Gateway (http://www.phylo.org); [76]. Bootstrapping was performed using a GTR plus Gamma nucleotide substitution model (-m GTRGAMMA ? 1000). The maximum likelihood tree was drawn using the ape package in $R$ [77]. The full tree including bootstrap confidence at the nodes is deposited in the Dryad Digital Repository (http://dx.doi.org/10.5061/dryad.3sh6d; [23]).

\section{Fluorescence in situ hybridization (FISH)}

FISH to mitotic chromosomes in larval neuroblasts was performed as previously described [78,79]. Briefly, brains were dissected from 3rd instar larvae and fixed in 1.8\% paraformaldehyde, $45 \%$ acetic acid. Fixed brains were denatured at $95 \mathrm{C}$ and hybridized overnight at $30 \mathrm{C}$. Slides were washed in $4 \mathrm{X}$ SSCT three times, and 0.1X SSC three times before mounting in Vectashield with DAPI. The probe was a biotinylated, nick translated PCR product specific to Rsp repeats $D$. melanogaster (F-5 GG AAAATCACCCATTTTGATCGC and R-5 CCGAATT CAAGTACCAGAC for D. melanogaster FISH) or Rsp-like repeats in D. sechellia (F-5 ACTGATTATCATCGCCTGGT and R-5 TCCAGTTCGCCTGGTAGTTT; for D. sechellia, D. simulans, and D. mauritiana FISH).

\section{Availability of supporting data}

The data sets supporting the results of this article are available in the Dryad repository, [doi:10.5061/dryad. 3sh6d and http://dx.doi.org/10.5061/dryad.3sh6d] and GenBank (KP016744-KP016746).

\section{Additional file}

Additional file 1: Table S1. BACs used in this paper. Table S2. Repeat comparison between BACs mapping to $U$ and $3 \mathrm{~L}$ (80C-D) and h39. Reported are pairwise percent identity (\%ID) and 95\% confidence intervals (95\% C.I.). Table S3. Percent identity between consensus Rsp, Rsp-like and RIX repeats. Figure $\mathbf{S} 1$. Maximum likelihood tree showing relationship between Rsp and Rsp-like repeats the melanogaster group. Figure S2. FISH with digested, unmapped BAC maps to both $2 R$ and $3 L / 3 R$ in mitotic chromosomes from brains. Figure $\mathbf{S} 3$. Alignment of heterochromatic $R s p$ family repeats (canonical Rsp of D. melanogaster and Rsp-like of the simulans clade, D. erecta and D. yakuba repeats) and euchromatic RIX repeats in species of the melanogaster subgroup.

\section{Abbreviations}

WGS: Whole genome shotgun; NGS: Next generation sequencing; Rsp: Responder; Rsp-like: Responder-like; RIX: Responder-like on X; SD: Segregation distorter; BAC: Bacterial artificial chromosome; FISH: Fluorescence in situ hybridization.

\section{Competing interests}

The author declares that she has no competing interests.

\section{Acknowledgements}

Id like to thank Daven Presgraves for helpful discussions throughout the course of this work, anonymous reviewers and Mohamed Noor with Axios Review for helpful comments, D. Emerson Khost for pointing out Rsp-like repeat unit length, and Anthony Geneva for help with RaxML and ape. This work was supported by an NIH-NRSA fellowship (5F32GM105317-02) from the National Institute of General Medical Sciences to A.M.L. and a David and Lucile Packard Foundation grant to Daven C. Presgraves.

Received: 21 October 2014 Accepted: 5 November 2014

Published online: 25 November 2014

\section{References}

1. Orgel LE, Crick FH: Selfish DNA: the ultimate parasite. Nature 1980, 284(5757):604 607

2. Doolittle WF, Sapienza C: Selfish genes, the phenotype paradigm and genome evolution. Nature 1980, 284(5757):601 603

3. Ohno S: So much junk DNA in our genome. Brookhaven Symp Biol 1972 23:366 370 .

4. Szybalski W: Use of cesium sulfate for equilibrium density gradient centrifugation. Methods Enzymol 1968, 12B:330 360

5. Yunis JJ, Yasmineh WG: Heterochromatin, satellite DNA, and cell function. Structural DNA of eucaryotes may support and protect genes and aid in speciation. Science 1971, 174(4015):1200 1209.

6. Sandler L, Novitski E: Meiotic drive as an evolutionary force. Am Nat 1957 . 91(857):105 110

7. Sandler L, Hiraizumi Y, Sandler I: Meiotic drive in natural populations of Drosophila melanogaster. I. the cytogenetic basis of segregationdistortion. Genetics 1959, 44(2):233 250.

8. Pimpinelli S, Dimitri P: Cytogenetic analysis of segregation distortion in Drosophila melanogaster: the cytological organization of the Responder (Rsp) locus. Genetics 1989, 121(4):765 772

9. Wu Cl, Lyttle TW, Wu ML, Lin GF: Association between a satellite DNA sequence and the Responder of Segregation Distorter in D. melanogaster. Cell 1988, 54(2):179 189. 
10. Larracuente AM, Presgraves DC: The selfish Segregation Distorter gene complex of Drosophila melanogaster. Genetics 2012, 192(1):33 53.

11. Houtchens K, Lyttle TW: Responder (Rsp) alleles in the Segregation Distorter (SD) system of meiotic drive in Drosophila may represent a complex family of satellite repeat sequences. Genetica 2003, 117(2 3):291 302.

12. Moschetti R, Caizzi R, Pimpinelli S: Segregation distortion in Drosophila melanogaster: genomic organization of Responder sequences. Genetics 1996, 144(4):1365 1371.

13. McAllister BF, Werren JH: Evolution of tandemly repeated sequences: What happens at the end of an array? J Mol Evol 1999, 48(4):469 481.

14. Kuhn GC, Kuttler H, Moreira-Filho O, Heslop-Harrison JS: The 1.688 repetitive DNA of Drosophila: concerted evolution at different genomic scales and association with genes. Mol Biol Evol 2012, 29(1):7 11.

15. Eickbush TH, Eickbush DG: Finely orchestrated movements: evolution of the ribosomal RNA genes. Genetics 2007, 175(2):477 485

16. Smith GP: Evolution of repeated DNA sequences by unequal crossover. Science 1976, 191(4227):528 535.

17. Dover G: A Molecular drive through evolution. Bioscience 1982, 32(6):526 533

18. Dover $\mathrm{G}$ : Concerted evolution, molecular drive and natural selection. Curr Biol 1994, 4(12):1165 1166

19. Elder JF Jr, Turner BJ: Concerted evolution of repetitive DNA sequences in eukaryotes. Q Rev Biol 1995, 70(3):297 320.

20. Liao D: Concerted evolution: molecular mechanism and biological implications. Am J Hum Genet 1999, 64(1):24 30

21. Hoskins RA, Smith CD, Carlson JW, Carvalho AB, Halpern A, Kaminker JS, Kennedy C, Mungall CJ, Sullivan BA, Sutton GG, Yasuhara JC, Wakimoto BT, Myers EW, Celniker SE, Rubin GM, Karpen GH: Heterochromatic sequences in a Drosophila whole-genome shotgun assembly. Genome Biol 2002, 3(12):RESEARCH0085.

22. Caizzi R, Caggese C, Pimpinelli S: Bari-1, a new transposon-like family in Drosophila melanogaster with a unique heterochromatic organization. Genetics 1993, 133(2):335 345.

23. Larracuente AM: Data from: the organization and evolution of the Responder satellite in species of the Drosophila melanogaster group: dynamic evolution of a target of meiotic drive. Dryad Digital Repository. doi:10.5061/dryad.3sh6d.

24. Cabot EL, Doshi P, Wu ML, Wu Cl: Population genetics of tandem repeats in centromeric heterochromatin: unequal crossing over and chromosomal divergence at the Responder locus of Drosophila melanogaster. Genetics 1993, 135(2):477 487

25. Temin RG, Ganetzky B, Powers PA, Lyttle TW, Pimpinelli S, Wu C-I, Hiraizumi Y: Segregation Distorter (SD) in Drosophila melanogaster: genetic and molecular analysis. Am Nat 1991, 137:287 331.

26. Osoegawa K, Vessere GM, Li Shu C, Hoskins RA, Abad JP, de Pablos B, Villasante $A$, de Jong PJ: BAC clones generated from sheared DNA. Genomics 2007, 89(2):291 299.

27. Hoskins RA, Carlson JW, Kennedy C, Acevedo D, Evans-Holm M, Frise E, Wan KH, Park S, Mendez-Lago M, Rossi F, Villasante A, Dimitri P, Karpen GH, Celniker SE: Sequence finishing and mapping of Drosophila melanogaster heterochromatin. Science 2007, 316(5831):1625 1628

28. Clark AG, Eisen MB, Smith DR, Bergman CM, Oliver B, Markow TA, Kaufman TC, Kellis M, Gelbart W, lyer VN, Pollard DA, Sackton TB, Larracuente AM, Singh ND, Abad JP, Abt DN, Adryan B, Aguade M, Akashi H, Anderson WW, Aquadro CF, Ardell DH, Arguello R, Artieri CG, Barbash DA, Barker D, Barsanti P, Batterham P, Batzoglou S, Begun D, et al: Evolution of genes and genomes on the Drosophila phylogeny. Nature 2007, 450(7167):203 218.

29. Britten RJ, Kohne DE: Repeated sequences in DNA. Hundreds of thousands of copies of DNA sequences have been incorporated into the genomes of higher organisms. Science 1968, 161(3841):529 540.

30. Hatch FT, Mazrimas JA: Fractionation and characterization of satellite DNAs of Kangaroo Rat (Dipodomys-Ordii). Nucleic Acids Res 1974, 1(4):559 575

31. Ugarkovic D, Petitpierre E, Juan C, Plohl M: Satellite DNAs in Tenebrionid species - structure, organization and evolution. Croat Chem Acta 1995, 68(3):627 638

32. Zhu Q, Pao GM, Huynh AM, Suh H, Tonnu N, Nederlof PM, Gage FH, Verma IM: BRCA1 tumour suppression occurs via heterochromatin-mediated silencing. Nature 2011, 477(7363):179 184

33. Zhang P: A trans-activator on the Drosophila $Y$ chromosome regulates gene expression in the male germ line. Genetica 2000, 109:141 150.

34. Pezer Z, Brajkovic J, Feliciello I, Ugarkovic D: Transcription of satellite DNAs in insects. Prog Mol Subcell Biol 2011, 51:161 178.
35. Lemos B, Araripe LO, Hartl DL: Polymorphic Y chromosomes harbor cryptic variation with manifold functional consequences. Science 2008 319(5859):91 93.

36. Hughes SE, Gilliland WD, Cotitta JL, Takeo S, Collins KA, Hawley RS: Heterochromatic threads connect oscillating chromosomes during prometaphase I in Drosophila oocytes. PLoS Genet 2009, 5(1):e1000348.

37. He B, Caudy A, Parsons L, Rosebrock A, Pane A, Raj S, Wieschaus E: Mapping the pericentric heterochromatin by comparative genomic hybridization analysis and chromosome deletions in Drosophila melanogaster. Genome Res 2012, 22(12):2507 2519.

38. Ferree PM, Barbash DA: Species-specific heterochromatin prevents mitotic chromosome segregation to cause hybrid lethality in Drosophila. PLoS Biol 2009, 7(10):e1000234.

39. Dernburg AF, Sedat JW, Hawley RS: Direct evidence of a role for heterochromatin in meiotic chromosome segregation. Cell 1996, 86(1):135 146.

40. Csink AK, Henikoff S: Something from nothing: the evolution and utility of satellite repeats. Trends Genet 1998, 14(5):200 204

41. Chippindale AK, Rice WR: Y chromosome polymorphism is a strong determinant of male fitness in Drosophila melanogaster. Proc Natl Acad Sci U S A 2001, 98(10):5677 5682.

42. Cattani MV, Presgraves DC: Incompatibility between $\mathrm{X}$ chromosome factor and pericentric heterochromatic region causes lethality in hybrids between Drosophila melanogaster and its sibling species. Genetics 2012, 191(2):549 559.

43. Brennecke J, Aravin AA, Stark A, Dus M, Kellis M, Sachidanandam R, Hannon GJ: Discrete small RNA-generating loci as master regulators of transposon activity in Drosophila. Cell 2007, 128(6):1089 1103.

44. Bayes JJ, Malik HS: Altered heterochromatin binding by a hybrid sterility protein in Drosophila sibling species. Science 2009, 326(5959):1538 1541.

45. Pezer Z, Ugarkovic D: RNA Pol II promotes transcription of centromeric satellite DNA in beetles. PLOS ONE 2008, 3(2):e1594.

46. Sun X, Wahlstrom J, Karpen G: Molecular structure of a functional Drosophila centromere. Cell 1997, 91(7):1007 1019

47. Henikoff S, Ahmad K, Malik HS: The centromere paradox: stable inheritance with rapidly evolving DNA. Science 2001, 293(5532):1098 1102.

48. Walker PM: Origin of satellite DNA. Nature 1971, 229(5283):306 308

49. Hoskins RA, Nelson CR, Berman BP, Laverty TR, George RA, Ciesiolka L, Naeemuddin M, Arenson AD, Durbin J, David RG, Tabor PE, Bailey MR, DeShazo DR, Catanese J, Mammoser A, Osoegawa K, de Jong PJ, Celniker SE, Gibbs RA, Rubin GM, Scherer SE: A BAC-based physical map of the major autosomes of Drosophila melanogaster. Science 2000, 287(5461):2271 2274.

50. Gemayel $R$, Vinces $M D$, Legendre $M$, Verstrepen $\mathrm{KJ}$ : Variable tandem repeats accelerate evolution of coding and regulatory sequences. Annu Rev Genet 2010, 44:445 477

51. Hiraizumi $Y$, Nakazima K: Deviant sex ratio associated with segregation distortion in Drosophila melanogaster. Genetics 1967 55(4):681 697

52. Kataoka Y: A genetic system modifying segregation distortion in a natural population of Drosophila melanogaster in Japan. Jap J Genet 1967, 42:327 337.

53. Hiraizumi Y, Thomas AM: Suppressor systems of Segregation Distorter (SD) Chromosomes in Natural Populations of Drosophila melanogaster. Genetics 1984, 106(2):279 292.

54. Hiraizumi Y, Albracht JM, Albracht BC: X-linked elements associated with negative segregation distortion in the SD system of Drosophila melanogaster. Genetics 1994, 138(1):145 152.

55. Gell SL, Reenan RA: Mutations to the piRNA pathway component aubergine enhance meiotic drive of segregation distorter in Drosophila melanogaster. Genetics 2013, 193(3):771 784.

56. Tao $Y$, Araripe L, Kingan SB, Ke Y, Xiao H, Hartl DL: A sex-ratio meiotic drive system in Drosophila simulans. Il: an X-linked distorter. PLoS Biol 2007, 5(11):e293.

57. Pezer Z, Ugarkovic D: Transcription of pericentromeric heterochromatin in beetles satellite DNAs as active regulatory elements. Cytogenet Genome Res 2009, 124(3 4):268 276

58. Carone DM, Longo MS, Ferreri GC, Hall L, Harris M, Shook N, Bulazel KV, Carone BR, Obergfell C, O'Neill MJ, O'Neill RJ: A new class of retroviral and satellite encoded small RNAs emanates from mammalian centromeres. Chromosoma 2009, 118(1):113 125.

59. Saito K, Nishida KM, Mori T, Kawamura Y, Miyoshi K, Nagami T, Siomi H, Siomi MC: Specific association of Piwi with rasiRNAs derived from retrotransposon and heterochromatic regions in the Drosophila genome. Gene Dev 2006, 20(16):2214 2222 
60. Powers PA, Ganetzky B: On the components of segregation distortion in Drosophila melanogaster. V Molecular analysis of the Sd locus. Genetics 1991, 129(1):133 144.

61. Presgraves DC, Gerard PR, Cherukuri A, Lyttle TW: Large-scale selective sweep among Segregation Distorter chromosomes in African populations of Drosophila melanogaster. PLoS Genet 2009, 5(5):e1000463.

62. Levinson G, Gutman GA: Slipped-strand mispairing: a major mechanism for DNA sequence evolution. Mol Biol Evol 1987, 4(3):203 221.

63. Ugarkovic D, Plohl M: Variation in satellite DNA profiles causes and effects. EMBO J 2002, 21(22):5955 5959.

64. Garrigan D, Kingan SB, Geneva AJ, Andolfatto P, Clark AG, Thornton KR, Presgraves DC: Genome sequencing reveals complex speciation in the Drosophila simulans clade. Genome Res 2012, 22(8):1499 1511.

65. Temin RG, Marthas M: Factors influencing the effect of segregation distortion in natural populations of Drosophila melanogaster. Genetics 1984, 107(3):375 393.

66. Adams MD, Celniker SE, Holt RA, Evans CA, Gocayne JD, Amanatides PG, Scherer SE, Li PW, Hoskins RA, Galle RF, George RA, Lewis SE, Richards S, Ashburner M, Henderson SN, Sutton GG, Wortman JR, Yandell MD, Zhang Q Chen LX, Brandon RC, Rogers YH, Blazej RG, Champe M, Pfeiffer BD, Wan KH, Doyle C, Baxter EG, Helt G, Nelson CR, et al: The genome sequence of Drosophila melanogaster. Science 2000, 287(5461):2185 2195.

67. Li H, Durbin R: Fast and accurate long-read alignment with BurrowsWheeler transform. Bioinformatics 2010, 26(5):589 595.

68. Kearse M, Moir R, Wilson A, Stones-Havas S, Cheung M, Sturrock S, Buxton S, Cooper A, Markowitz S, Duran C, Thierer T, Ashton B, Meintjes P, Drummond A: Geneious Basic: an integrated and extendable desktop software platform for the organization and analysis of sequence data. Bioinformatics 2012, 28(12):1647 1649.

69. Hu TT, Eisen MB, Thornton KR, Andolfatto P: A second-generation assembly of the Drosophila simulans genome provides new insights into patterns of lineage-specific divergence. Genome Res 2013, 23(1):89 98.

70. Mackay TF, Richards S, Stone EA, Barbadilla A, Ayroles JF, Zhu D, Casillas S, Han Y, Magwire MM, Cridland JM, Richardson MF, Anholt RR, Barron M, Bess C, Blankenburg KP, Carbone MA, Castellano D, Chaboub L, Duncan L, Harris Z, Javaid M, Jayaseelan JC, Jhangiani SN, Jordan KW, Lara F, Lawrence F, Lee SL, Librado P, Linheiro RS, Lyman RF, et al: The Drosophila melanogaster Genetic Reference Panel. Nature 2012, 482(7384):173 178.

71. Langmead B, Salzberg SL: Fast gapped-read alignment with Bowtie 2. Nat Methods 2012, 9(4):357 359.

72. Li H, Handsaker B, Wysoker A, Fennell T, Ruan J, Homer N, Marth G, Abecasis G, Durbin R: The Sequence Alignment/Map format and SAMtools. Bioinformatics 2009, 25(16):2078 2079.

73. Simpson JT, Wong K, Jackman SD, Schein JE, Jones SJ, Birol I: ABySS: a parallel assembler for short read sequence data. Genome Res 2009, 19(6):1117 1123

74. Huelsenbeck JP, Ronquist F: MRBAYES: Bayesian inference of phylogenetic trees. Bioinformatics 2001, 17(8):754 755.

75. Stamatakis A: RAxML-VI-HPC: maximum likelihood-based phylogenetic analyses with thousands of taxa and mixed models. Bioinformatics 2006, 22(21):2688 2690

76. Miller MA, Pfeiffer W, Schwartz T: Creating the CIPRES Science Gateway for inference of large phylogenetic trees. In Proceedings of the Gateway Computing Environments Workshop (GCE): November 14, 2010. New Orleans, LA: IEEE; 2010:1 8 .

77. Paradis E, Claude J, Strimmer K: APE: analyses of phylogenetics and evolution in R language. Bioinformatics 2004, 20(2):289 290.

78. Larracuente AM, Noor MA, Clark AG: Translocation of Y-linked genes to the dot chromosome in Drosophila pseudoobscura. Mol Biol Evol 2010, 27(7):1612 1620

79. Larracuente AM, Ferree PM: Simple method for fluorescence DNA in situ hybridization to 2-D chromosomes. JoVE. in press.

doi:10.1186/s12862-014-0233-9

Cite this article as: Larracuente: The organization and evolution of the Responder satellite in species of the Drosophila melanogaster group: dynamic evolution of a target of meiotic drive. BMC Evolutionary Biology 2014 14:233

\section{Submit your next manuscript to BioMed Central and take full advantage of:}

凶Convenient online submission

$\otimes$ Thorough peer review

$\otimes$ No space constraints or color $₫$ gure charges

$\otimes I m m e d i a t e$ publication on acceptance

Q Inclusion in PubMed, CAS, Scopus and Google Scholar

$\otimes$ Research which is freely available for redistribution

Submit your manuscript at www.biomedcentral.com/submit
C Biomed Central 